\title{
USING E-MAIL FOR SOCIAL AND DOMESTIC PURPOSES
}

\author{
Processes, practices and attitudes
}

\author{
Kathy Buckner and Mark Gillham \\ Deptartment of Information Management, Queen Margaret University College, Edinburgh, \\ United Kingdom.
}

Key words: E-mail, CMC, Conventional mail, Social network analysis.

\begin{abstract}
A graphical conversational prop is used along with interviews to elicit the processes, practices and attitudes of twelve home based social e-mail users to this and alternative communication methods. Contact frequency of e-mail communication resembles telephone usage more than face-to-face or letter; the text quantity of e-mails tends to be less variable than posted communications; e-mail usage seems less time dependent than telephone use. While there is considerable variation in attitudes to social communication by both e-mail and by letter, e-mail is found by many to be more spontaneous, to encourage shorter, less formal and more immediate communication, and by some to be of lower quality and value.
\end{abstract}

\section{INTRODUCTION}

This research project explores the effect that e-mail, through home based connection to the Internet, has on the social interaction between people in the domestic situation. In previous studies we have examined the effect of computing in the home environment. Specific issues identified related to: positioning of computers within private or communal spaces; using technology for working and learning at home; gender and power; the effect increasing use of computers has on the time available for other family activities; and, facilitation and participation in shared activities through the computer [5]. It is only in the last few years of the 1990s that personal use of e-mail is starting to reach a critical mass where it is becoming a significant 
method of social communication [12]. E-mail and its effects needs to be studied in respect to other communication methods which are mediated by communication technology (telephone, fax), by a physical delivery system (post) or without intermediaries, that is, through face-to-face contact [19], [13]. However in this paper we particularly focus on comparing the processes and practices adopted by people using those media types which use the written word to support social communication, that is, e-mail and letters sent and received on paper through a postal system.

The rapid growth and decreasing cost of the use of networked technologies for personal communication through the internet (in the UK $46 \%$ have a PC at home or work [17]) and mobile phones (41\% of people in the UK have a mobile phone [10]) is now facilitating real choice for many in relation to which mode of communication is used to maintain and foster links with friends and family. Wellman et al [18] and Haythornthwaite [8], discuss the way in which the use of a range of media types for the exchange of different kinds of information supports both work and friendship ties with, for example, e-mail facilitating frequent, informal contact between individuals. On-line networks which are established using newsgroups, listservers or discussion lists to support a particular activity [14] not only create a sense of community between participants but may lead to the establishment of one-to-one correspondence between participants and may as a consequence extend an individual's social network. The nature and social use of the Internet is explored by others (e.g. Maignan and Lukas [11]). Casalegno [4] in his study of the Blacksburg Electronic Village notes that listservers are used for "creating ties between otherwise separate individuals" and that "people use e-mail to correspond daily with friends and family".

Much relevant research has previously been undertaken in the fields of work and learning. Within the work domain Åsterbro [1] discusses how management interventions and staff social interactions impact on the uptake of new technologies. Ngwenyama and Lee [15] analyse the way in which meaning is conveyed in e-mail correspondence in a work situation. Zach [19] explores the context in which particular communication modes are selected and the effectiveness of the subsequent communication. Networked learning studies report on the value of technology to support students working both on and off campus by providing communication networks for those with similar needs and concerns (e.g. Buckner and Morss [3]). In addition to supporting specific learning activities, students use the network connections provided to maintain, extend and establish their social networks.

In our work we are primarily investigating the way in which the scope of existing social networks may be enhanced or developed through the use of e-mail. We compare the process of using e-mail with the process of using 
conventional mail. We examine the quantifiable characteristics of e-mail communication and how it compares with other communication forms in respect of a) frequency of communication, both in absolute terms and relative to other forms, b) quantity of information exchanged (measured by time of verbal exchanges and physical quantity of written material) and, c) the time of day when communication takes place.

We look in depth at the nature of e-mail exchanges and examine the mechanisms involved in e-mail communication. We also compare the nature of the content of e-mail and letter examining issues of formality, intimacy, style of writing and language.

\section{METHODOLOGY}

Twelve people were obtained for the project by convenience sampling. All had to have a computer in their home that was connected to the Internet with e-mail capabilities, and, also use e-mail for social purposes. The participants lived in Edinburgh or within a 10 miles radius of the city. Data was collected during the summer of 1999 during a visit from the researcher lasting between one and two hours.

In the field of social network analysis Grosser [6] has undertaken work in relation to the charting and analysis of social cohesion and the graphical display of cognate groups that are key components of the study of group dynamics. Social network analysis has also been used as a tool for the study of information exchange [7]. In this study we used a conversational prop, SO-grams (Significant Other-grams), developed as part of the work undertaken on the Living Memory project (EU ESPRIT project 25621) to elicit maps of the social networks of a number of people who use e-mail for social and domestic purposes. The process of constructing SO-grams has been reported elsewhere [2]. They are diagrams with the subject name placed in a circle at the centre of the page; acquaintances with their level of significance indicated by different sizes circle; frequency of contact indicated by three different coloured or different thicknesses of line; and the media used for social contact indicated by symbols representing conventional mail (letters and cards), telephone, face-to-face, fax and e-mail. Using this technique followed by a structured interview has enabled us to gain insight into the patterns of behaviour of our subjects and to compare their use of e-mail with their use of conventional mail. 


\subsection{General data}

Demographic information was requested about the person as well as details of the make up of the household. Background questions were asked about the participant's experience of owning and using computers, about the main uses of the computer by the participant and other household members and about its location within the home. Data was gathered on the use of email and other computer mediated communication tools by the interviewee and others within the household.

\subsection{Communication patterns}

From the contacts shown in the SO-gram the participants were asked to select up to six with whom they had some e-mail contact, where possible selecting people from each of the significance (circle size) categories. For each of these selected contacts we recorded a) the frequency of each type of communication, b) the relative quantity of each communication type c) the typical lengths of communications of each type (in time or space) and, d) the usual time(s) of day each communication type is used. Data were also collected on the frequency of use of e-mail (by themselves and other members of the household), Internet discussion lists, the WWW and Internet chat rooms.

\section{$2.3 \quad$ Mechanics of e-mail use}

Participants were questioned in some detail about how they work with email in terms of receiving, sending and storing messages. Comparisons were made with the processes employed with conventional mail and e-mail. The relationships with other household members were investigated.

\subsection{Attitudes to e-mail and conventional mail}

The relative advantages and disadvantages of e-mail and conventional mail were discussed in particular reference to composing messages, sending and receiving. Feelings about both forms of communication were investigated.

The procedure was piloted on one participant before starting with the main sample. 


\section{RESULTS AND DISCUSSION}

\subsection{Demographics and experience with computers}

The sample consisted of five women and seven men. Ages ranged from 15 to over 50, with the 50+ category with the most (4). All but one participant was in employment or full time education and those in work were mainly in professional employment. For just under half (5) their current computer was the first in the household while the rest had previously owned computers. All had used computers (at work or home) for three years or more, with a mean length of use of 12.8 years.

Most participants (10) had Intel or compatible processor based desktop personal computers running a version of the MS Window operating system, while the remainder owned Apple Macs. A variety of main reasons was given for getting the computer but their own, or someone else in the household's, needs to do work on it was the most common. Only two specifically mentioned e-mail as the main reason. The uses of the computer at home are specified as e-mail (all), word processing (7), WWW (6) and games, work and DTP ( 3 each). In all the 10 households with more than one person, others in the household used the computer.

\subsection{Communication patterns}

\subsubsection{E-mail use}

All the participants have been using e-mail for at least two years. Several have used it at work or college before doing so in the home. The average length of home use was approximately 2.5 years. Of the six households that had more than one e-mail user, only two shared e-mail addresses. It is apparent that there is often not a sharp divide between home and work when considering e-mail use. While eight of the 12 participants reported that they usually used e-mail for social communication from home, the other four mainly or equally did so from work.

\subsubsection{Frequency of different communication types}

There was a total of 61 selected contacts identified among the 12 participants in the study. In the analysis of communication with selected contacts note that all these contacts are in e-mail communication with the participants and are not necessarily representative of all the people they communicate with. Face-to-face, telephone and e-mail are the most frequently used media with letters/cards being sent with reasonable 
frequency (yearly or more) to under half of the selected contacts and fax used very rarely (Figure 1 ).

Most e-mail contacts (44/61) are described as being weekly or monthly. Face-to-face and telephone peak at seasonally/yearly and weekly/monthly respectively. The relatively high incidence of infrequent face-to-face contact would be expected as many of the chosen e-mail contacts live at a distance from the participants in the study.

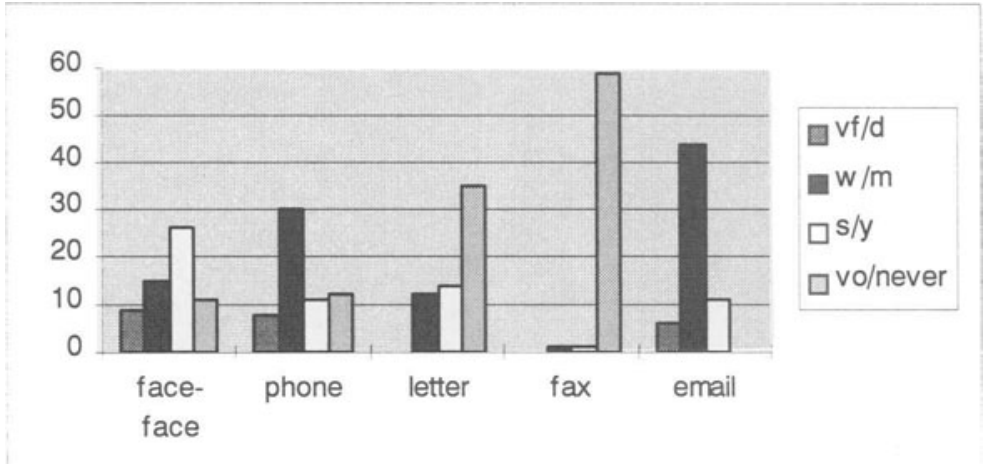

Figure 1. Frequency of use of different communication methods for selected contacts ${ }^{31}$.

\subsubsection{Quantity of letters and e-mail compared}

In terms of quantifiable content it is interesting to compare letters and email. Both these communication methods are asynchronous and predominantly text in contrast to the other main methods of face-to-face and telephone. A high proportion (83\%) of e-mails are from a few sentences to a few paragraphs in length (Figure 2). The length of letters has a wider spread with a surprising proportion of letters of several pages (33.3\%), and the same proportion of just one or a few sentences. The latter are perhaps mainly made up of cards.

31 Explanation of key in Figure 1: vf/d = very frequent: several times a day/about every day or two; $\mathrm{w} / \mathrm{m}=$ weekly (includes once very few days - to once every week)/monthly (includes once very 2 weeks to once every two months); s/y = seasonally (several times a year: between 2 and 6 times a year)/ once a year; Vo/never = very occasionally: less than once a year/never 


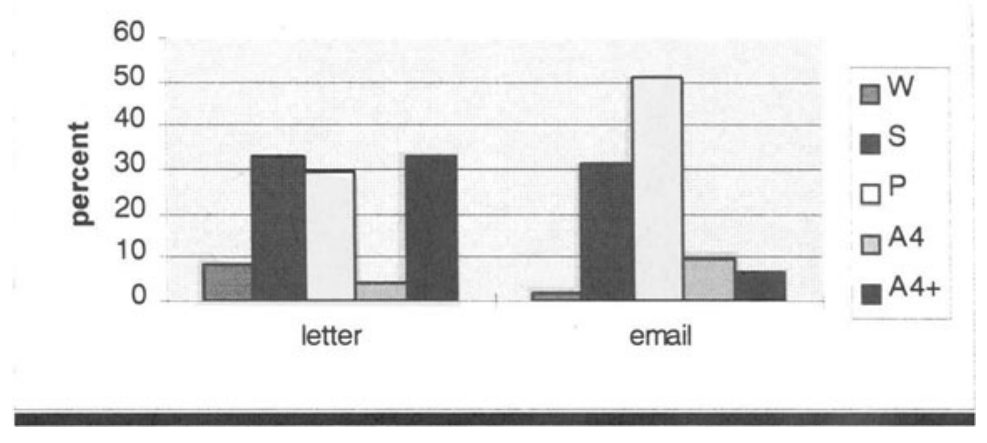

Figure 2. Comparison of the estimated amount of text sent in letters and e-mail ${ }^{32}$

\subsubsection{Usage time of telephone and e-mail compared}

To help understand usage behaviour participants estimated the time of day when they undertook the different communication activities. Communication by e-mail was more variable than use of the telephone which peaked in the evening (59\%). The greater flexibility of e-mail in part may be reflected by social e-mail use at work or college where the same cost penalties as the telephone are not relevant. It may also be because home online access through most ISPs (Internet Service Providers) is at local call rates.

\subsection{Communication processes and practices}

The benefits of the immediacy of e-mail as a medium becomes apparent when we study the behaviour of participants when they compose, send and receive messages. We compare their behaviour with e-mail with their approach to using conventional mail.

\subsubsection{Composing messages}

When writing messages almost all the participants compose messages directly into the e-mail system although a third sometimes word process (particularly if the message is lengthy) and then load into the mail application. Attachments, mostly photographs and word processed letters,

32 Explanation of key in Figure 2: $\mathrm{W}=\mathrm{a}$ few words, a phrase; $\mathrm{S}=$ one or a few sentences; $\mathrm{P}=$ one or a few paragraphs; $\mathrm{A} 4=$ about a page (A4 or equivalent); $\mathrm{A} 4+=$ several pages. The number of contacts to whom letters are sent $=24$, e-mail is sent to all 61 of the selected contacts (a criterion for selection). 
are sent by $75 \%$ of our sample. A few subjects (3) compose joint messages mostly to family members. Just over half the sample thought that the process of composing e-mail was the same or similar to that of composing conventional mail. Some felt that the e-mail was more spontaneous than letter writing:

"I tend to think less and then edit more when e-mailing".

"Letters take more consideration, deliberation and time"

\subsubsection{Receiving and responding to messages}

E-mail, much like conventional mail, is for some people exciting to receive. Just as checking the doormat for post might be the first thing some of us do in the morning, so checking for e-mail is often the first activity after switching on the PC. There is some evidence from our research that some users get a 'technological buzz' from using e-mail.

All our subjects read their e-mail directly off the screen, there being no concern expressed about the privacy of this form of display of personal correspondence, and with only four sometimes printing messages out. It is surprising that with most ISPs now operating at local call rates, eight subjects still download e-mails and read them off-line. Most (9) respond immediately to messages received and when they don't respond immediately they all respond very quickly, either by the next day (5) or within a day or two (5). Half the sample felt that e-mail decreased the time taken to respond to correspondence when compared with conventional mail.

\subsubsection{Storing correspondence}

Once a message has been dealt with all except one subject remove it from their default mail box. Three subjects delete the messages straight away while the remainder devise different filing systems. These mostly involve creating folders for different friends or groups of friends; one involves categorisation by year; one creates content categories (e.g. technical messages, social messages) and 2 people organise messages by the actions they have taken e.g. Sent, Read, Deleted, Retained. For some their filing systems are very similar to the method they use for conventional mail, however, for others it is a radically different process e.g. while all conventional mail is kept - sometimes for very long periods (up to 10 years in one case)-all e-mail is deleted. It is interesting to compare these filing systems with the one used by Charles Dodgson (Lewis Carroll, 1832-1898) to record his personal correspondence. He recorded all items sent and 
received in chronological order, each with its own reference number and with different symbols used to denote a) the type of item (e.g. parcel, telegram, letter) and b) if action is required [16]. Our subjects' technologically enabled filing systems automate most of these features and facilitate greater, and arguably better, organisation of messages, although it is clear that as with traditional filing systems some are better at using them than others.

\subsection{Attitudes towards using e-mail compared with conventional mail}

Speed and convenience are seen as the main benefits of using e-mail. Cost is not a decisive factor in making a choice about which medium to use for correspondence. The immediacy of e-mail combined with its ease of use (no need to buy or find a stamp and envelope, no need to go to a post office or queue) outweigh any consideration of cost difference.

A third of the sample feel that messages sent by e-mail are shorter than those sent by conventional mail although this is not necessarily viewed as a disadvantage and by some is perceived as a positive effect of the use of the electronic medium. Messages tend to be viewed as more informal, sometimes at the expense of perceived quality. The ability to write and then edit if necessary was a distinct advantage for some (3). One person indicated that they "Use e-mail as a supplement to telephone conversations". This reinforces the work undertaken by Haythornthwaite which suggests that multiple modes of communication tend to be used when ties with friends and acquaintances are stronger [8].

All participants have received word-processed attachments and all but one has received pictures or multimedia files. This sharing of artefacts (mostly photographs (8) - of which four were specifically of family members) also helps to strengthen the ties and social bond between individuals [8]. Some participants expressed a preference for receiving conventional mail. As one subject states:

"I prefer receiving letters. Nothing to do with the effort expended (or lack of it) - [I] prefer the physical element of a conventional letter, not the same as printing it off."

Leslie in Wired magazine puts this rather poetically another way: 
"whoever has printed out a pleasing e-mail message discovers that on the page it is rendered lifeless like an exquisite tropical fish removed from its tank" [9]

Nevertheless, the immediacy and convenience of e-mail still seem to be such positive benefits that they outweigh the lack of receipt of a physical artefact. So as one of our subject stated:

"E-mail is not merely supplemental, it complements phone calls and face-to-face conversations. It is fundamental to work for many people and may become so on a social level."

\section{CONCLUSION}

Use of e-mail as a social communication medium is a very personal activity. Individuals adapt its use in a way which suits them and their way of life. Contact frequency of e-mail communication resembles telephone usage more than face-to-face or letter; the text quantity of e-mails tends to be less variable than posted communications; e-mail usage seems less time dependent than telephone use. Our study provided a large amount of data about our subjects' perception of the nature and future use of e-mail and we will report on this elsewhere. In future studies we aim to focus on in-depth observation and analysis of individual behaviour with e-mail in the social and domestic setting.

\section{REFERENCES}

[1] Åsterbro, T. (1995). The effect of management and social interactions on the intra-firm diffusion of electronic mail systems. IEE Transactions on Engineering Management 42(4), 319-331.

[2] Davenport, E. \& Buckner, K., (1998) SO-grams: a personal visualisation toolkit for intranet users, In Zimmermann, H.H. \& Schramm, V. (Eds.) Knowledge management and Kommunicationssystem, Proceedings des 6 Internationalen Symposiums fur Inforamtionswissenschaft (ISI 98), (pp 67-79), UVK Universitatsverlag Konstanz GmbH: Konstanz.

[3] Buckner, K., \& Morss K., (1999). The importance of task appropriateness in computer supported collaborative learning. ALT-J, 7(1), 33-38.

[4] Casalegno, F. (1999). Community networking and social interaction in the wired city of Blacksburg. In Stathis, K. (Ed.), Local nets: Proceedings of the international workshop on community-based interactive systems (pp.13-23). London: Imperial College of Science and Technology. 
[5] Davenport, E., Higgins, M., \& Gillham, M., (1996). Designing a probe to explore home information systems in the United Kingdom. Online and CD-ROM Review 20(2), 75-79.

[6] Grosser, K. (1991). Human networks in organizational information processing. In M. Williams (Ed.) Annual Review of Information Science and Technology, 26, pp.349ff. Medford, NJ: Learned Information Inc.

[7] Haythomthwaite, C. (1996). Social network analysis: an approach and technique for the study of information exchange. LISR, 18, 323-342.

[8] Haythornthwaite, C. (1999). A social network theory of tie strength and media use: a framework for evaluating multi-level impacts of new media, October 10 1999, http://alexia.lis.uiuc.edu/ haythom/sna_theory.html. Accessed 17/12/1999.

[9] Leslie, J. (1994). Mail Bonding. Wired 2.03, March, http://www.wired.com/wired/archive/2.03/e-mail.html. Accessed 12/1/2000.

[10] Lorenz, A. (2000). Sunday Times: Business Focus, 9 January 2000, p5.

[11] Maignan, I., \& Lukas, B.A. (1997). The nature and social uses of the internet: a qualitative investigation. Journal of Consumer Affairs, 31(2), 346-371.

[12] Markus, M.L., (1990). Towards a "critical mass" theory of interactive media. In Fulk, J., \& Steinfield, C.W., Organisations and Communication Technology (pp.194-218). Newbury Park, CA: Sage.

[13] Michailidis, A., \& Rada, R., (1997). Activities and communication modes. International Journal of Human-Computer Studies, 46(4), 469-483.

[14] Millen, D., \& Dray, S., Information sharing in an online community of journalists. In Buckner, K. (Ed.), Esprit i3 workshop on ethnographic studies in real and virtual environments: inhabited information spaces and connected communities, (pp.53-60). Edinburgh: Queen Margaret University College.

[15] Ngwenyama, O.K., \& Lee, A.S., (1997). Communication richness in electronic mail: critical social theory and the contextuality of meaning. MIS Quarterly: Management Information Systems, 21(2), 145-167.

[16] Stratton, J., (1999). Eight or nine wise words about letter writing 5. On registering correspondence. FireBlade Coffeehouse, http://www.hoboes.com $/ \mathrm{html} /$ FireBlade /Carroll/Words/Letters5.html.Accessed: 12/1/2000.

[17] Technology Tracker (1999). September, e-Mori, http://www.e-public.co.uk /factfigures.html. Accessed 10/1/2000.

[18] Wellman, B., Salaff, J., Dimitrova, D., Garton, L., Gulia, M., \& Haythornthwaite, C., (1996). Computer networks as social networks: Collaborative work, telework, and virtual community. Annual Review of Sociology, 22, 213-238.

[19] Zach, M.H., (1994). Electronic messaging and communication effectiveness in an ongoing work group. Information and Management, 26(4) 231-241. 